\title{
Morphological Operations on 3D and 4D Images: From Shape Primitive Detection to Skeletonization
}

\author{
Pieter P. Jonker \\ Pattern Recognition Group, Faculty of Applied Sciences, \\ Delft University of Technology, \\ Lorentzweg 1, 2628 CJ Delft,The Netherlands \\ pieter@ph.tn.tudelft.nl
}

\begin{abstract}
This paper describes a practical approach to mathematical morphology and ways to implement its operations. The first chapters treat a formalism that has the potential of implementing morphological operations on binary images of arbitrary dimensions. The formalism is based on sets of structuring elements for hit-or-miss transforms whereas each structuring element actually describes a shape primitive. The formalism is applied to two and three dimensional binary images and the paper includes structuring elements for topology preserving thinning or skeletonization and various skeleton variants. The generation of shape primitive detecting masks is treated as well as their application in segmentation, accurate measurement and conditions for topology preserving. The formalism is expanded to four-dimensional images and elaborates on the extension of $3 \mathrm{D}$ skeletonization to $4 \mathrm{D}$ skeletonization. A short excursion was made to methods based on 3D and 4D Euler - cluster count methods.
\end{abstract}

\section{Introduction}

In this paper a practical introduction to Mathematical Morphology applied on binary images of arbitrary dimensions is presented. Mathematical Morphology applied on binary images is also known as Cellular Logic Processing. Whereas the Mathematical Morphology sprouts from a mathematical domain, Cellular Logic Processing has its roots in binary image processing on massively parallel Cellular Logic Machines. The approaches are, however, similar and have their own charm. The method and formalism as described have been developed in parallel with the design of an architecture for real-time image processing [1] $]$. However, the method is (also) implemented in software. In the next chapter, the use of sets of structuring elements is explained and operations in $2 \mathrm{D}$ and $3 \mathrm{D}$. In chapter three, the connectivity paradox and topology breaking properties are studied for $\mathrm{N}$ dimensional images, followed by skeletonization using shape primitives. The generation and verification of shape primitives is explained, and in chapter four, their extension to the fourth dimension. 


\section{Sets of Structuring Elements}

\subsection{Hit-or-Miss Transformations}

The field of cellular logic image processing and mathematical morphology is extensively described in several works (Golay 1969 [2]; Preston 19 [3]; Serra 19\$2 [4], $19 \$ 8$ [5], Giardina 19\$8 [6], Heijmans 1994 [7], Soille 1999 [8], and many others). A basic operation in mathematical morphology is the Hit-or-Miss transformation, and as a starting point for this section we will quote its definition by Serra (1982):

The Hit-or-Miss transformation is a point-by-point transformation of a set $X$, that is performed in the following way:

Choose and fix a structuring element $S$, the datum of $S$ being two sets $S^{1}$ and $S^{2}$. Suppose $S$ is centered at the point $x$ of $X$, denoted by $S=\left(S_{x}^{1}, S_{x}^{2}\right)$, then a point belongs to the Hit-or-Miss transformation $Y \leftarrow X \otimes S$ of $X$, if and only if $S_{x}^{1}$ is included in $X$ and $S_{x}^{2}$ is included in the complement $X^{c}$ of $X$, or:

$$
Y \leftarrow X \otimes S \equiv\left\{x \mid\left(S_{x}^{1} \subset X, S_{x}^{2} \subset X^{c}\right)\right\}
$$

To show the similarities, this definition in the mathematical domain will be mirrored to the domain of binary image processing with its convolution kernel-like approach, by associating an origin and a vector structure with it.

We associate with the universe -the union of $X$ and $X^{c}$-, a square tessellated image $\mathrm{X}$ of size $\mathrm{m} \mathrm{x} \mathrm{n}$, and with the elements of the universe, the pixels of image $\mathrm{X}$. We can then associate with the set $X$, the foreground of image $\mathrm{X}$ and with the set $X^{c}$ its background. Likewise for the sets $Y$ and $Y^{c}$, and image Y. As structuring element $S$ consists of two disjunct sets $S^{1}$ and $S^{2}$, then its universe is formed by the union of the disjunct sub-sets $S^{1}, S^{2}$ and $S^{c}, S^{c}$ being the complement of the union of $S^{1}$ and $S^{2}$. We associate with the union of $S^{1}, S^{2}$ and $S^{c}$, a square tessellated image $\mathrm{S}$ of size ix $\mathrm{j}$, and with the elements of $S^{1}, S^{2}$ and $S^{c}$, the pixels in the image $\mathrm{S}$. Then we can associate with the set $S^{1}$ the foreground pixels, with the set $S^{2}$ the background pixels, with the union of $S^{1}$ and $S^{2}$ the do-care pixels and with the set $S^{c}$ the don't care pixels. In our case of binary image processing, the foreground is defined to have the value TRUE or 1, the background to have the value FALSE or 0 , and the don't cares to have the value D. Hence, don't cares are used to implement the unrestricted set $S$ with image $\mathrm{S}$ having a fixed size and shape.

Let $\mathrm{x}, \mathrm{y}$ and $\mathrm{s}$ be the elements (pixels) of $\mathrm{X}, \mathrm{Y}$ and $\mathrm{S}$, and let the size of S e.g. be $\mathrm{i}=$ $\mathrm{j}=3$, then the $3 \times 3$ structuring element $\mathrm{S}$ consists of $\mathrm{s}^{0}, \mathrm{~s}^{1}, \ldots \mathrm{s}^{8}$ the east (E), north-east $(\mathrm{NE})$, north $(\mathrm{N}), \ldots \ldots$. , south-east (SE) neighbours of the central (C) pixel $\mathrm{s}^{8}$ itself, and an equivalent $3 \times 3$ neighbourhood $M_{k}$ around pixel $x_{k}$, consists of $x_{k}^{0}, x_{k}^{1} \ldots . x_{k}^{7}$ : the $\mathrm{E}, \mathrm{NE}, \mathrm{N}, \ldots \ldots . . \mathrm{SE}$ neighbours of the pixel $\mathrm{x}_{\mathrm{k}}$ and $\mathrm{x}_{\mathrm{k}}$ the central $(\mathrm{C})$ pixel $\mathrm{x}_{\mathrm{k}}$ itself. 
The point-by-point transformation $Y \leftarrow X \otimes S$ can now be implemented for binary image processing with the neighbourhood transformation $\mathrm{Y} \leftarrow \mathrm{X} \cong \mathrm{S}$, or $\left\{\forall \mathrm{k}: \mathrm{y}_{\mathrm{k}} \leftarrow \mathrm{M}_{\mathrm{k}} \cong \mathrm{S}\right\}$ with $\mathrm{M}_{\mathrm{k}}$ and $\mathrm{S}$ centered around pixel $\mathrm{x}_{\mathrm{k}}$, and with $\cong$ the symbol for an inexact match. Informally, the inexact neighbourhood matches $\left\{\forall \mathrm{k}: \mathrm{y}_{\mathrm{k}} \leftarrow \mathrm{M}_{\mathrm{k}} \cong \mathrm{S}\right\}$ can be described as follows:

If for any pixel $\mathrm{x}_{\mathrm{k}}$ in an input image $\mathrm{X}$, its neighbourhood $\mathrm{M}_{\mathrm{k}}$ matches inexactly with a structuring element $\mathrm{S}$, the pixel $\mathrm{y}_{\mathrm{k}}$ of output image $Y$ is set to one. If $\mathrm{M}_{\mathrm{k}}$ does$n^{\prime}$ t match $\mathrm{S}, \mathrm{y}_{\mathrm{k}}$ is set to zero.

In the inexact neighbourhood match the foreground pixels in $\mathrm{S}$ should match with foreground pixels in $\mathrm{M}_{\mathrm{k}}$ at the same positions AND the background pixels in S should match with background pixels in $\mathrm{M}_{\mathrm{k}}$ at the same positions, whereas in the don't care positions of $\mathrm{S}$ a match is not required, or:

$$
\mathrm{y}_{\mathrm{k}} \leftarrow \stackrel{8}{\wedge}\left\{\mathrm{x}_{\mathrm{k}=0}^{\mathrm{i}} \cong \mathrm{s}^{\mathrm{i}}\right\} \text { with: }
$$

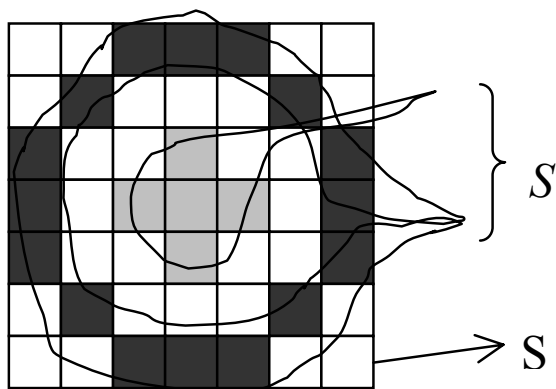

\begin{tabular}{|l|l|l}
\hline $\mathrm{x}$ & $\mathrm{s}$ & $(\mathrm{x} \cong \mathrm{s})$ \\
\hline 0 & 0 & 1 \\
\hline 0 & 1 & 0 \\
\hline 1 & 0 & 0 \\
\hline 1 & 1 & 1 \\
\hline 1 & $\mathrm{D}$ & 1 \\
\hline 0 & $\mathrm{D}$ & 1
\end{tabular}

Fig. 1. Structuring element $S$ and its implementation with an image $\mathrm{S}$ with fixed size and shape

We now extend this inexact neighbourhood match in two ways, first to:

$$
\mathrm{y}_{\mathrm{k}} \leftarrow \underset{\mathrm{i}=1}{\mathrm{p}}\left\{\mathrm{M}_{\mathrm{k}} \cong \mathrm{S}_{\mathrm{i}}^{\mathrm{S}}\right\}
$$

or: If for any pixel $\mathrm{x}_{\mathrm{k}}$ in an input image $\mathrm{X}$, its neighbourhood $\mathrm{M}_{\mathrm{k}}$ matches one mask $\mathrm{S}_{\mathrm{i}}^{\mathrm{S}}$ from a set of masks $\mathrm{S}^{\mathrm{S}}$ the pixel $\mathrm{y}_{\mathrm{k}}$ of output image $Y$ is set to one, else to zero.

Meaning that the union of all mask matches is taken.

And further to: $\quad y_{k} \leftarrow\left\{\underset{i=1}{\vee v}\left\{M_{k} \cong S_{i}^{S}\right\}\right\} \vee\left\{\underset{i=1}{\vee v}\left\{\overline{M_{k} \cong S_{i}^{R}}\right\}\right\}$ 
or: If for any pixel $\mathrm{x}_{\mathrm{k}}$ in an input image $\mathrm{X}$, its neighbourhood $\mathrm{M}_{\mathrm{k}}$ matches any mask $\mathrm{S}_{\mathrm{i}}^{\mathrm{S}}$ from a given set of masks $\mathrm{S}^{\mathrm{S}}$ the pixel $\mathrm{y}_{\mathrm{k}}$ of output image $\mathrm{Y}$ is set to one, else set to zero, OR if its neighbourhood matches any mask $\mathrm{S}_{\mathrm{i}}^{\mathrm{R}}$ from a given set of masks $\mathrm{S}^{\mathrm{R}}$, the pixel $\mathrm{y}_{\mathrm{k}}$ is set to zero, else to one.

The image transformation $\mathrm{Y} \leftarrow \mathrm{X} \cong \mathrm{S}$ is now implemented with a set of masks $\mathrm{S}$ as structuring element, consisting of a subset $S^{S}$ (the SET-masks) and a subset $S^{R}$ (the RESET-masks), one of which may be empty. This means that either a pixel $y_{k}$ is set to zero, if one of the RESET masks fits, or the pixel is set to one, if one of the SET mask fits, where the SET masks are chosen to dominate over the RESET masks.

Finally, a second input image $\mathrm{Z}$ can be used to locally enable/disable the transformation, yielding the dyadic operation $\mathrm{Y} \leftarrow f(\{\mathrm{X}, \mathrm{Z}\} \cong \mathrm{S})$, or:

$$
\mathrm{y}_{\mathrm{k}} \leftarrow\left(\left\{\underset{\mathrm{i}=1}{\mathrm{v}}\left\{\mathrm{M}_{\mathrm{k}} \cong \mathrm{S}_{\mathrm{i}}^{\mathrm{S}, \mathrm{z}}\right\}\right\} \vee\left\{\underset{\mathrm{i}=1}{\mathrm{v}}\left\{\overline{\mathrm{M}_{\mathrm{k}} \cong \mathrm{S}_{\mathrm{i}}^{\mathrm{R}, \mathrm{z}}}\right\}\right\}\right)
$$

If a "mask-bit" $\mathrm{Z}$ of a mask of the set $\mathrm{S}$ is set don't care, the transformation is enabled. If $\mathrm{Z}$ is do-care, then if the pixel $\mathrm{z}_{\mathrm{k}}$ of $\mathrm{Z}$ matches with $\mathrm{Z}$, the operation is disabled, else enabled. Operations that use $Z$ to locally mask-off or seed operations, are the propagation operation and the anchor-skeleton.

Note that the term structuring element is used in broad sense. We will also use the word kernel, mask or mask set (all indicated with S) to indicate a structuring element. In many cases, a mask set can be transformed into a set with a single mask, and vice versa, without effecting its functional properties. This will be demonstrated in the sequel. A theoretical treatment of the manipulation of sets of structuring elements, finding the minimal set (the basis) and deriving the equivalent set that is spanned by scanning an image with a sequence of structuring elements, can be found e.g. in the article of Jones and Svalbe (1994)[9], which starts from Matheron's theorem (1975) [10] that "a simple algebra of union intersection and translation suffices to implement all general translation invariant and increasing set mappings".

An operation on an image $\mathrm{X}$ can be done by performing the Hit-or-Miss transformation with a mask set $\mathrm{S}$ once (6), twice or more (7), or infinite (8: the n-pass form):

$$
\begin{gathered}
\mathrm{Y} \leftarrow(\mathrm{X} \cong \mathrm{S}) \\
\mathrm{Y} \leftarrow(((\mathrm{X} \cong \mathrm{S}) \cong \mathrm{S}) \cong \mathrm{S}) \ldots . \mathrm{S}) \\
\mathrm{X}_{0} \leftarrow \mathrm{X} ;\left\{\forall \mathrm{i}: \mathrm{X}_{\mathrm{i}+1} \leftarrow\left(\mathrm{X}_{\mathrm{i}} \cong \mathrm{S}\right)\right\} ; \mathrm{Y} \leftarrow \mathrm{X}_{\infty}
\end{gathered}
$$

In the latter case an iterative scheme is used, which can be implemented by transforming the image until it is idempotent under the transformation. 
In many cases practical use can be made of the intermediate results in the output image. This is called (spatial) recursion. For instance, if the transformation is performed by a raster scan over the image, i.e. from top-left to bottom-right, the fact that the neighbours NE, N, NW, W of the pixel $y_{k}$ have already obtained a new value can be utilized. For this purpose, the neighbourhood $\mathrm{M}_{\mathrm{k}}$ of pixel $\mathrm{x}_{\mathrm{k}}^{\mathrm{i}}$ will be extended by $\mathrm{y}_{\mathrm{k}}^{\mathrm{r} 1}, \mathrm{y}_{\mathrm{k}}^{\mathrm{r} 2}, \mathrm{y}_{\mathrm{k}}^{\mathrm{r} 3}, \mathrm{y}_{\mathrm{k}}^{\mathrm{r} 4}$, the newly obtained values and the structuring element $\mathrm{S}$ will be extended by: $\mathrm{S}^{\mathrm{rl}}, \mathrm{S}^{\mathrm{r} 2}, \mathrm{~S}^{\mathrm{r} 3}, \mathrm{~S}^{\mathrm{r} 4}$.

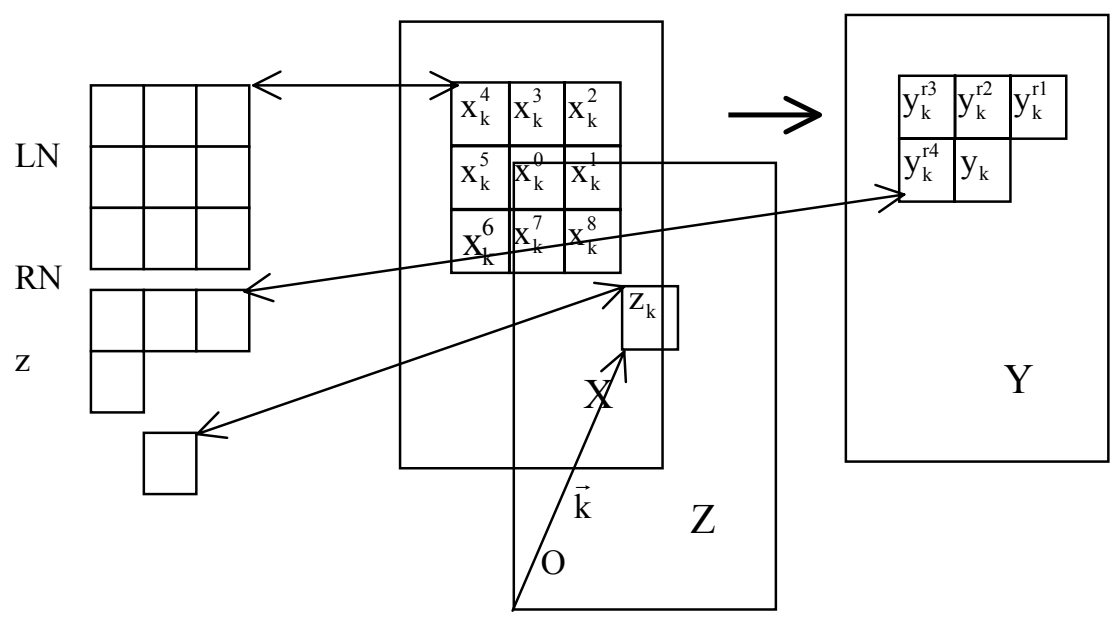

Fig. 2. Drawing conventions for $\mathrm{Y} \leftarrow f(\{\mathrm{X}, \mathrm{Y}, \mathrm{Z}\} \cong \mathrm{S})$ Here shown for $2 \mathrm{D}$ images and $3 \mathrm{x}$ 3 neighbourhoods. The LN is taken from input image $\mathrm{X}$, the $\mathrm{RN}$ from output image Y. For dyadic operations a second input image $\mathrm{Z}$ is used

So now both the Local Neighbourhood (LN: the original value $\mathrm{x}_{\mathrm{k}}$ and its neighbourhood $\mathrm{x}_{\mathrm{k}}^{0}, \mathrm{x}_{\mathrm{k}}^{1}, \mathrm{x}_{\mathrm{k}}^{2}, \ldots . \mathrm{x}_{\mathrm{k}}^{7}$ ), and the Recursive Neighbourhood ( $\mathrm{RN}$ : the newly obtained values $\left.\mathrm{y}_{\mathrm{k}}^{\mathrm{r1}}, \mathrm{y}_{\mathrm{k}}^{\mathrm{r} 2}, \mathrm{y}_{\mathrm{k}}^{\mathrm{r} 3}, \mathrm{y}_{\mathrm{k}}^{\mathrm{r} 4}\right)$, can be used in the neighbourhood matching procedure. The class of cellular logic operations performed with this extended neighbourhood will be referred to as recursive neighbourhood operations (RNO) in contrast with the local neighbourhood operations (LNO) that use the normal neighbourhood only. Note that both classes have a dyadic form for which $S$ is extended with an element $z$ and $M_{k}$ with a pixel $z_{k}$ of a second image $Z$, yielding:

$$
\mathrm{Y} \leftarrow f(\{\mathrm{X}, \mathrm{Y}, \mathrm{Z}\} \cong \mathrm{S})
$$

Figure 2 pictures the matching process with a single mask from a set $\mathrm{S}$, whereas the neighbourhood $\mathrm{M}_{\mathrm{k}}$ is extracted from the three different images. Note that the Recursive Neighbourhood that can be realised is implementation dependent, and that when using a software raster scan over the image, it is beneficial for RNOs to scan in all odd 
scans from top-left to bottom-right and in all even scans from bottom-right to top-left. In this case, the RN should be transposed to match with the scan direction.

\subsection{Basic Morphological Operations in $\mathrm{X}_{\mathrm{N}}$}

The most frequently used known Local Neighbourhood Operations are: Erosion, Dilation, Contour extraction, Spot noise removal (pepper and salt removal) and Majority vote (binary rank filtering). The two most frequently used recursive and iterative cellular logic operations are: Connected component extraction or Propagation and Topology Preserving Thinning or Skeletonisation. Figures 3 and 4 show some structuring elements in the form of mask-sets for simple operations in $\mathrm{X}_{2}$ and $\mathrm{X}_{3}$.

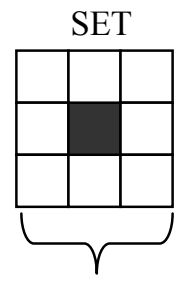

Copy

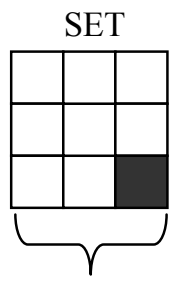

Move NE

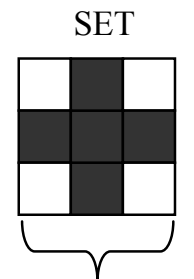

Erode 8cet

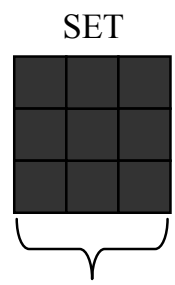

Erode $4 \mathrm{cct}$
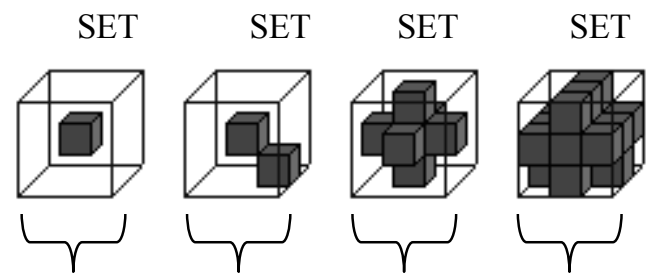

SET

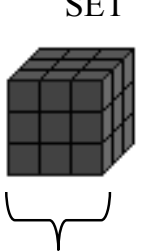

Copy Move NE Erode 26cet Erode 18'cct Erode 6cc1

Fig. 3. Structuring elements for simple operations in $X_{2}$ and $X_{3}$

The structuring elements in Figure 3 are LNOs based on single masks of the SET type, meaning that the central element is set to foreground if the $3^{\mathrm{N}}$ neighbourhood matches, else to background.

The operations for $\mathrm{X}_{2}$ are: copy the image, move the image to North-East, erode an 8 connected contour from all objects in the image, erode a 4 connected contour from all objects in the image. Note that the "erode 8cct mask" is in fact a template that matches on foreground area in $\mathrm{X}_{2}$, it does neither match on background nor places where it touches the contour of an object in any of the directions N,E,S,W. Erode 4cct does the same but in addition will not match on pixels in the NE, NW, SE, SW directions. Because of the template matching aspect of the masks, the principle can be expanded to $\mathrm{X}_{\mathrm{N}}$ as the masks for $\mathrm{X}_{3}$ show.

Figure 4 shows the dilation operation: It comprises a mask that matches on background area in $X_{2}$ and on background volume in $X_{3}$ while not touching objects. Where 
the mask matches, the background is reset to background, where the mask does not match, the background is set to foreground. From the contour detection mask set in $\mathrm{X}_{2}$ and $\mathrm{X}_{3}$ the first mask matches on foreground not touching the object contour, which is reset to background, and the second mask matches on all background which is also reset to background. Both masks do not match on the object contours, which will hence be set to foreground. A result is shown for $X_{3}$, where on a cubic volume with a ball shaped hole, de 26 connected contour surface was detected using the maskset "detect 26cct". Note that for a better view of the result, the cube was cut open short behind its front face. Finally, the mask-set for Pepper and Salt removal for $\mathrm{X}_{2}$ shows that the first mask repairs the pepper and the second mask repairs the salt noise.

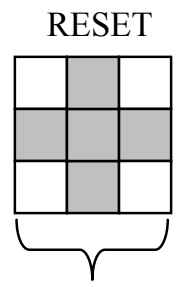

Dilate $4 \mathrm{cct}$

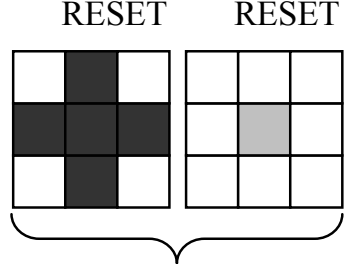

Detect $8 \mathrm{cct}$

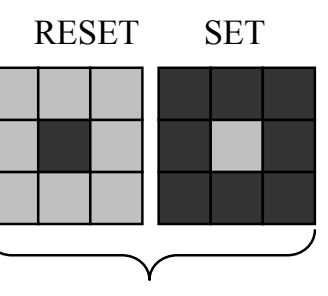

Spot noise removal

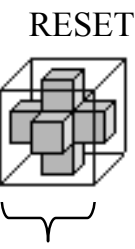

Dilate $26 \mathrm{cct}$

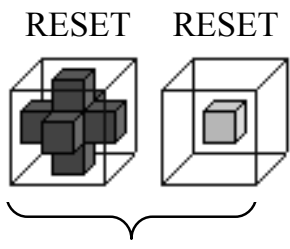

Detect $26 \mathrm{cct}$

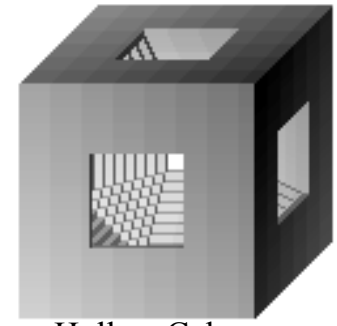

Hollow Cube

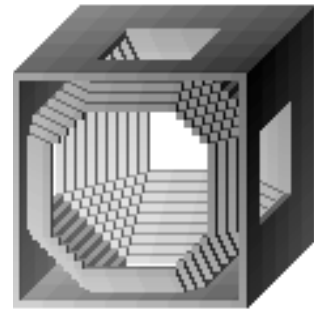

Detected 26cct

Fig. 4. RESET masks, masks sets and mixed SET / RESET sets. Opaque elements are don't cares, light grey are background elements and dark grey are foreground elements

The relation between the SET and RESET forms of the mask-sets is given by the Boolean algebra, i.e. the theorem of De Morgan (1847)[11]. Defining mask sets using only SET conditions is possible, although in some cases this is not very parsimonious. For example, the transformation of dilate_4cct from a conjunctive canonical form to a disjunctive canonical form is given by:

$$
\begin{aligned}
& \overline{(\overline{\mathrm{C}} \wedge \overline{\mathrm{N}} \wedge \overline{\mathrm{W}} \wedge \overline{\mathrm{S}} \wedge \overline{\mathrm{E}})} \equiv(\mathrm{C} \vee \mathrm{N} \vee \mathrm{W} \vee \mathrm{S} \vee \mathrm{E}) \equiv \\
& (\overline{\mathrm{C}} \wedge \mathrm{N}) \vee(\overline{\mathrm{C}} \wedge \mathrm{W}) \vee(\overline{\mathrm{C}} \wedge \mathrm{S}) \vee(\overline{\mathrm{C}} \wedge \mathrm{E}) \vee(\mathrm{C})
\end{aligned}
$$

Dilate_4cct, first expressed as the "erosion of the background", expands to a set of five masks, expressing the dilation as: "copy the image in directions $\mathrm{N}, \mathrm{W}, \mathrm{S}, \mathrm{E}$ and take the union of all results and original image", and finally to a set of 5 masks that 
indicate that "any background pixel adjacent to the foreground is set to foreground". The discussion above leads us to the observation, that mask sets can be minimized by transforming them from the disjunctive canonical form to the conjunctive canonical form, or vice versa. And, that mask-sets can be manipulated using Boolean algebra and minimisation.

Figure 5 shows an example of a dyadic Recursive Neighbourhood Operation in $X_{2}$ and $X_{3}$; the propagation operation. Objects in an image are recursively dilated (the first mask), wherever foreground in image $\mathrm{Z}$ is found and background in $\mathrm{X}$ was found (the second mask).
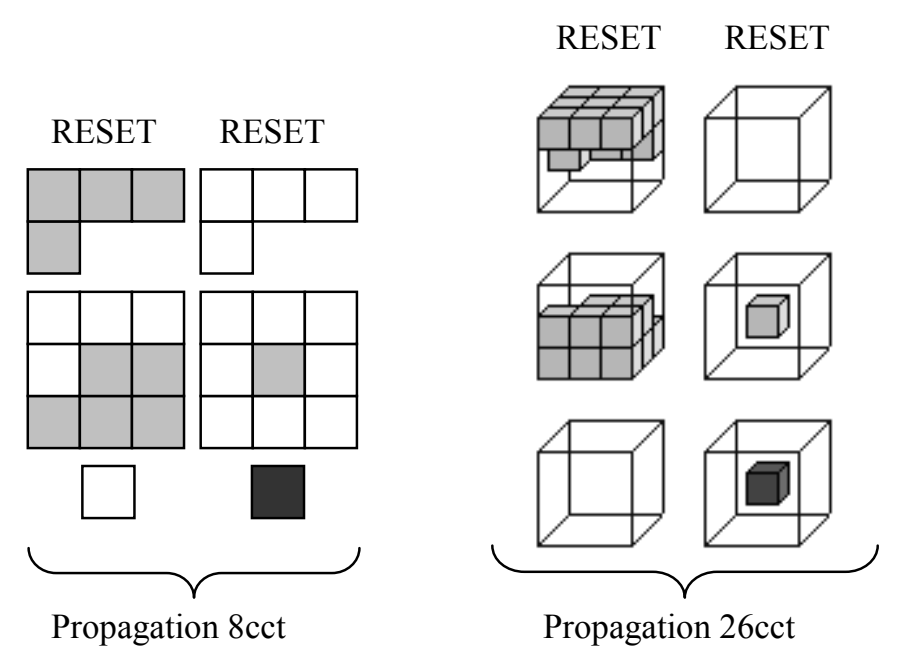

Fig. 5. Dyadic RN Operation in $X_{2}$ and $X_{3}$; the propagation operation

\subsection{Connectivities in $\mathbf{N}$ Dimensional Images $X_{N}$}

A set is connected if each pair of its point can be joined by a path along points that are all in the set. Within a square tessellated two-dimensional binary image $\mathrm{X}_{2}$ with objects on a background, there are two possibilities for the connectivity of object and background pixels. The objects can be chosen to consist of pixels connected with one or more of their 8 neighbours at (E, NE, N, NW, W, SW, S, SE) or they can be chosen to be 4-connected, with one or more of their neighbours at (E, N, W, S). See also Rosenfeldt and Pfaltz (1966) 12 for the connectivity paradox. This paradox extends to higher dimensions. Note that we define the connectivity of an object as the highest connectivity between any two adjacent elements within that object.

Tessellation in higher dimensions is also known as honeycombing. Coxeter [13 proves in his book on regular polytopes, that the only regular honeycomb (a single regular identical cell on a lattice) that exist in all dimensions $\mathrm{N}$ is the cubic honeycomb. In addition in 2D the tessellation with hexagons exist and in 4D two types of honeycombs exist (each-others dual), however, not with their vertices on a lattice. 
Although in 3D the Dodecahedron and Icosahedron exits, they cannot be used for honeycombing in 3D. C60 molecules or "bucky-balls" are truncated Icosahedrons, made from hexagons and pentagons. Above dimension 5 only the equivalents of the regular Tetrahedron, Octahedron and Cube exist as polytopes. Which leaves hypercubic honeycombing as the only way to extend our method to higher dimensions.

Let us define an $\mathrm{N}$-dimensional binary image $\mathrm{X}_{\mathrm{N}}$ as a bounded section of an equidistantly sampled Euclidean space of dimension $N$, with element values $\{0,1\}$. The elements of $X_{2}$ are referred to as pixels, the elements of $X_{3}$ as voxels, of $X_{4}$ and higher as hyper-voxels. Let $\mathrm{M}_{\mathrm{N}}^{\mathrm{n}}$ be an N-dimensional hyper-cubic neighbourhood with odd size $\mathrm{n}=2 \mathrm{k}+1$. For many morphological operations $3^{\mathrm{N}}$ neighbourhoods are used $(k=1, n=3)$, so $M_{N}^{n}$ is abbreviated to $M_{2}$ for a $3 \times 3$ and $M_{3}$ for a $3 \times 3 \times 3$ neighbourhood, etc. Connectivities can be derived by counting the number of image elements around a central element, within a hypersphere and within the neighbourhood. E.g. in $\mathrm{X}_{2}$ there are 4 pixels within radius 1 and 8 pixels within radius $\sqrt{2}$. In [1] and [14] it was shown that the connectivity between image elements can be derived by counting $\mathrm{E}$, the number of elements on a hyper sphere with radius $d$ around an image element that lie within the neighbourhood $\mathrm{M}_{\mathrm{N}}^{\mathrm{n}}$. E can be calculated by permuting the neighbourhood element co-ordinates, and is given by:

$$
E=\frac{N !}{\prod_{j=0}^{k}\left(n_{j} !\right)} 2^{\left(\mathrm{N}-\mathrm{n}_{0}\right)}
$$

From $\mathrm{E}$, we can derive $\mathrm{V}$, the total number of elements within the hyper-sphere within $\mathrm{M}_{\mathrm{N}}$ and hence the connectivity $\mathrm{G}=\mathrm{V}-1$. This yields as connectivities in a $3^{\mathrm{N}}$ neighbourhood for $\mathrm{X}_{2}:\{4,8\}$, for $\mathrm{X}_{3}:\{6,18,26\}$, for $\mathrm{X}_{4}:\{8,32,64,80\}$, etc., in a $5^{\mathrm{N}}$ neighbourhood for $X_{2}:\{12,20,24\}$, for $X_{3}:\{32,56,80,92,116,124\}$, etc.

\subsection{Connectivity Paradox in $\mathrm{X}_{3}$}

Connectivity rests on connected paths between pairs of points in a set. The paradox exists because with the sort of operations we do on the sets (like segmenting regions into objects) we demand that paths are boundaries. I.e. they cannot be crossed -nor tunneled- without breaking the path. In topology, the Jordan Curve theorem states that a simple closed curve lying in a plane divides the plane into precisely two regions and forms their common boundary. [15. This theorem extends to $X_{N}$. 


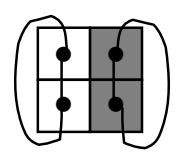

a

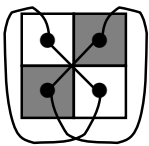

b

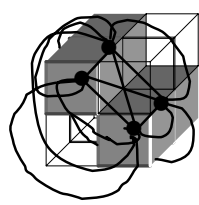

c

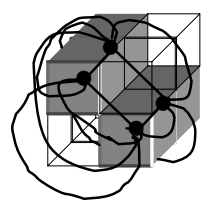

d

Fig. 6. Jordan Curves applied in $X_{2}$ and $X_{3}$

Consider a set of 4 points from a larger universe $\mathrm{X}_{2}$ with two sets each of two points (white and grey), see figure 6 . We will call this $2 \times 2$ neighbourhood a tile. Clearly when the grey set forms anywhere in the universe a closed boundary, incorporating the connection between the two grey points of the neighborhood, the white set cannot be connected. When we define that in a neighbourhood all points of a set directly adjacent to the boundary of the neighbourhood are assumed to be connected to any other point of the same set over that boundary, then for figure $6 \mathrm{~b}$ this means that if the grey set is connected, the white set cannot be connected. If we assume closure over the tile boundary, we can say that two points each at another side of a boundary cannot be connected. So we must choose, either white is face and edge connected and grey is only face connected, or vice versa.

We can extend this to higher dimensions, but then tiles are $2^{\mathrm{N}}$ neighbourhoods and closed boundaries are topologically equivalent to (hyper) spheres. Note that we consider connections over the tile boundaries now separately for each dimension. Figures $6 \mathrm{c}$ and $\mathrm{d}$ shows tiles in $\mathrm{X}_{3}$. Figure $6 \mathrm{c}$ is a topological equivalent of a sphere when we assume that the grey set is 26 connected and the white set is not. The white set must then be 6 connected. Figure $6 \mathrm{~d}$ is a topological equivalent of a sphere when we assume that the grey set is 18 connected and the white set not. The white set must then be 6 connected, while we don't care about the vertex connections. Vice versa, when we are sure that the white set is 6 connected, we might as well say that the grey set is 18 connected, as we don't care about the vertex connections. We can now make the observations that:

- The lowest (face) connectivity in any image $\mathrm{X}^{\mathrm{N}}$ is always a perfect boundary, it never leaks, all higher connectivities do. The reason is that a pair of face connected elements has unit extends perpendicular to its connection axis in all remaining dimensions.

- Usually we are interested in the properties of the foreground objects, the curvature of objects being one of them. The higher the connectivity, the smaller radii can be made, as can be imagined by observing the radii of 8 and 4 connected curves in $\mathrm{X}_{2}$ $(1, \sqrt{2})$ and 26,18 and 6 connected rings in $X_{3}(1, \sqrt{2}, \sqrt{3})$. If we assume that that the centers of the image elements represent sampling points of a physical phenomenon, then, with the same sampling density, smaller structures can be represented more accurately using a higher connectivity. This gives a preference for taking the highest possible connectivity as the foreground connectivity. 
Consequently: A good choice is to take the lowest connectivity as background as it never leaks and the highest connectivity as foreground as it provides us with the highest bending radius.

\section{Skeletonization in $X_{N}$}

In the previous sections, we have described a general principle for morphological operations on cubic tessellated binary images $X_{N}$, based on the scanning of the image with a set of masks that comprise the structuring element. The masks contain foreground, background and don't-cares. On each element of the image an inexact match between all masks of the set and the neighbourhood extracted from the image is performed, whereas the result written to the output image is the union of all matches.

So far, only erosion of foreground and background and matches on single elements (pixels, voxels, ...) have been used. Erosions can be described as a match on foreground area in $\mathrm{X}_{2}$ and on foreground volume in $\mathrm{X}_{3}$. In this chapter, skeletonization in $X_{N}$ is treated. Skeletonization can be seen as conditional erosion 16. Figure 7 shows that in $\mathrm{X}_{3}$ a volume is eroded to a curved surface -the surface skeleton-, where after the surface is eroded to a space curve -the curve skeleton-. Apparently, for skeletonization the condition for the erosion is that surfaces, or curves, should not be eroded. For those conditions, we need the notion of shape primitives in $\mathrm{X}_{\mathrm{N}}$.
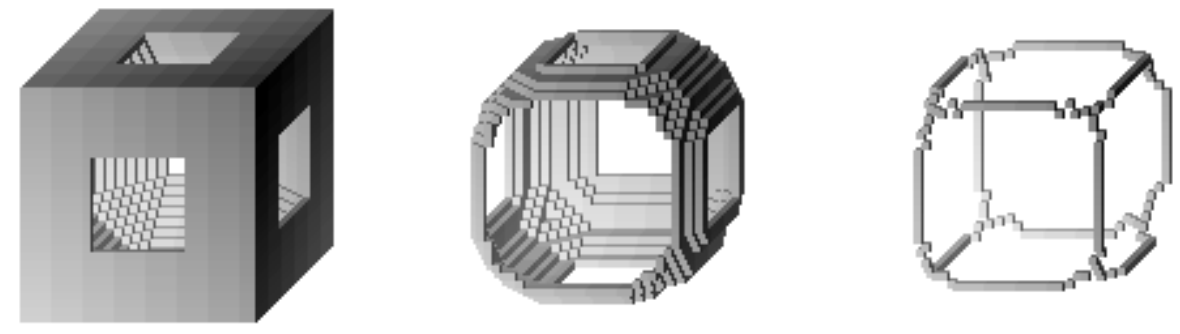

Fig. 7. Original, contour, surface-, and curve skeleton of a cube with hole in $X_{3}$

\subsection{Shape Primitives in $X_{N}$}

In $X_{N}$ we can identify shape primitives with a certain intrinsic dimension $\widetilde{N}$. For example, in $X_{2}$ we can distinguish points $(\widetilde{N}=0)$, curves $(\widetilde{N}=1)$ and surfaces $(\widetilde{N}$ $=2)$, in $X_{3}$ points, space curves, curved surfaces and flat volumes $(\widetilde{N}=3)$, and in $\mathrm{X}_{4}$ we can distinguish points, space curves, curved surfaces, curved volumes and flat 
hyper-volumes $(\widetilde{\mathrm{N}}=4)$. Objects in $\mathrm{X}_{\mathrm{N}}$ can now be considered as made of any arbitrary combination of shape primitives with intrinsic dimensions $(0 \leq \widetilde{N} \leq . N)$.

Figure 7 shows objects only consisting of volume, or surface, or space curve. It was already remarked that the $\left(6,18,26\right.$ connected) erosion conditions for $\mathrm{X}_{3}$ match on volumes except for the outer boundary of those volumes. Consequently, they are the shape primitive detectors for volumes in $\mathrm{X}_{3}$. The contour detection operations "Detect 6cct", "Detect 18'cct", and "Detect 26cct" were applied on a hollow volume (obtained by dilating a single voxel with 26 connected surface contours obtaining a sphere, and XOR-ing this with a cube). See figure 8.
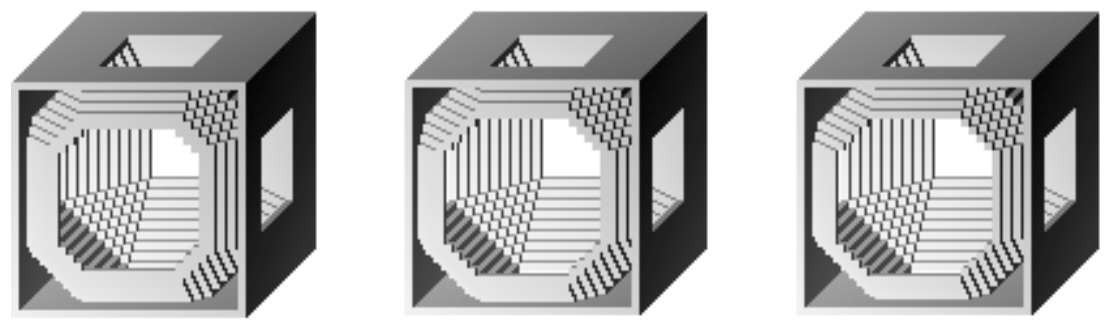

Fig. 8. 6, 18' and 26 connected surface contours in $X_{3}$

(The cube's front slices have been removed for better insight). All resulting surfaces prevent the leaking of 6 connected background. The first result shows a surface that is entirely made of face connected voxels, which prevents leaking of face, edge and vertex connected voxels. Therefore, the background is allowed to be 26 connected. The second result shows a surface that is entirely made of face connected voxels, which prevents leaking of face and edge connected background but allows leaking of vertex connected background. Therefore, the background is allowed to be 18 connected. The third result shows a surface that is entirely made of face and edge connected voxels, which allows leaking of edge and vertex connected background. So the background is should be face (6) connected.

In order to detect primitive shapes such as space curves, curved surfaces and volumes, we need shape primitives that match on all possible incarnations of those primitives shapes.

\subsection{The Generation of Shape Primitives in $X_{3}$}

Shape primitives for space curves can be found by remarking that an open (non circular) space curve in a $3^{3}$ neighbourhood consists of a central voxel and two mutually non touching neighbours. Figure 9 shows all possible configurations for 6 and 26 connected space curves generated by this rule. 

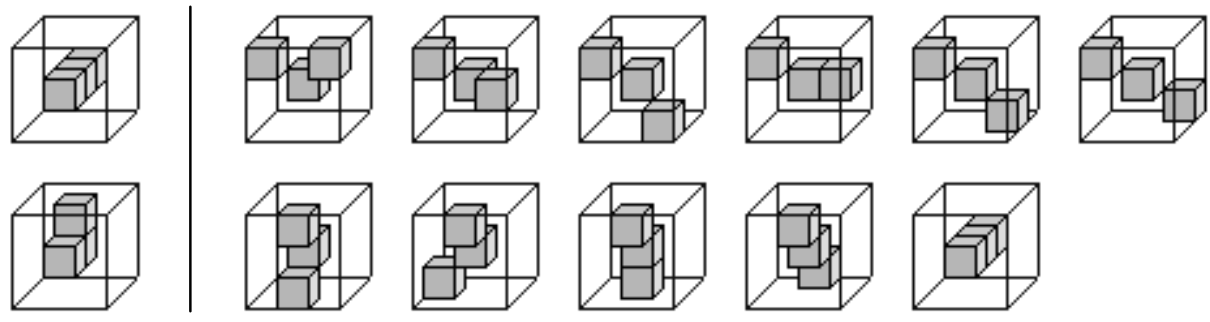

Fig. 9. The 6 connected space curve primitives and the 26 connected space curve primitives

Note that we omitted all rotated and mirrored versions and that non touching is defined for a certain connectivity. Hence, the second mask from the 6 connected set is not present in the 26 connected set. The set of 18 connected space curves is a subset of the 26 connected set.
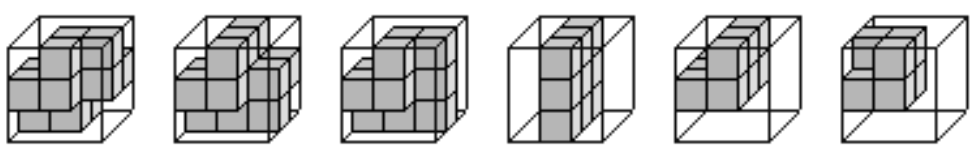

Fig. 10. The 6 Connected curved surface primitives

In a similar way, the surface primitives can be found. They can be generated by a central voxel encircled by a non-touching closed space curve. For a 6 connected surface set, the central voxel should be encircled by a 6 connected space curve (figure 10 ), for a 26 connected set by a 26 connected space curve (figure 11).
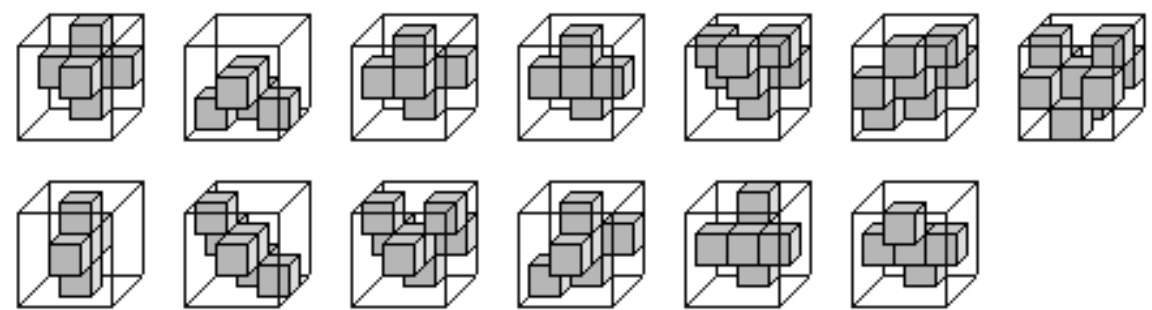

Fig. 11. The 26 connected curved surface primitives

Again non touching is defined for a certain connectivity. The generation procedure is a recursive search that generates all possible occasions. After this, all identical, mirrored and rotated variants are removed and the set is logically reduced to a minimal covering set [17]. Note when generating the surface primitives, their voxels stay in the 18 connected neighbourhood of the central voxel in both cases. The vertex connected positions of the central voxel are apparently don't care. Observe, that in the 6BG 26FG system, the space curves roam over the 26 positions, the curved surfaces over the 18 connected positions, and the voxels of the volume detection mask are found on the 6 connected positions. Consequently, we can state that in a 6BG-26FG system the space curves are 26 connected, the surfaces are 18 connected and the volumes are 6 
connected. In a 26BG-6FG system the space curves are 6 connected, the surfaces 18 connected and the volumes 26 connected.

If we have compound (bifurcating) objects, such as forking space curves, splitting surfaces, surfaces with sprouting curves etc., we must introduce don't cares. We can do this, because we can be more precise in our foreground-background paradox. We can state this paradox now as follows:

\section{A foreground curved surface is threatened to be pierced by a background space curve.}

2. A foreground space-curve is threatened to be broken by a background surface, breaking the connectivity of the curve.

We can now merge the sets. Rule 1 leads to figure 12, a merging of the sets from figures 11 and the first set of figure 9. Rule 2 leads to figure 13, a merging of the sets from figure 10 and the second set of figure 9. The set from figure 12 (when augmented with rotated and mirrored variants) can be used to identify any surface in an image $X_{3}$. The set of figure 13 can be used to identify any curve in an image $X_{3}$. For all the masks hold, that if the central voxel swaps its value, e.g. from foreground to background, the connectivity of the foreground is broken and the topology changes. As each mask hits individually, it can be tagged with a contribution to the length of a curve or to the area of a surface. Hence, surface area and curve length's of objects can be measured more accurately then simply counting voxe [s. 18].
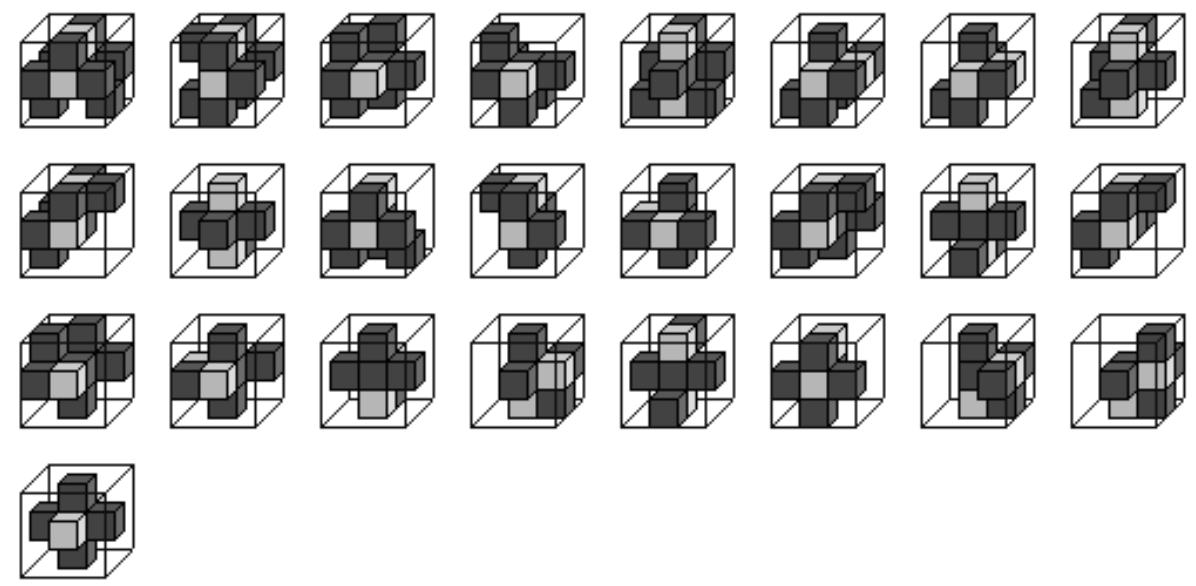

Fig. 12. Shape detectors for curved surfaces "Surf26" 

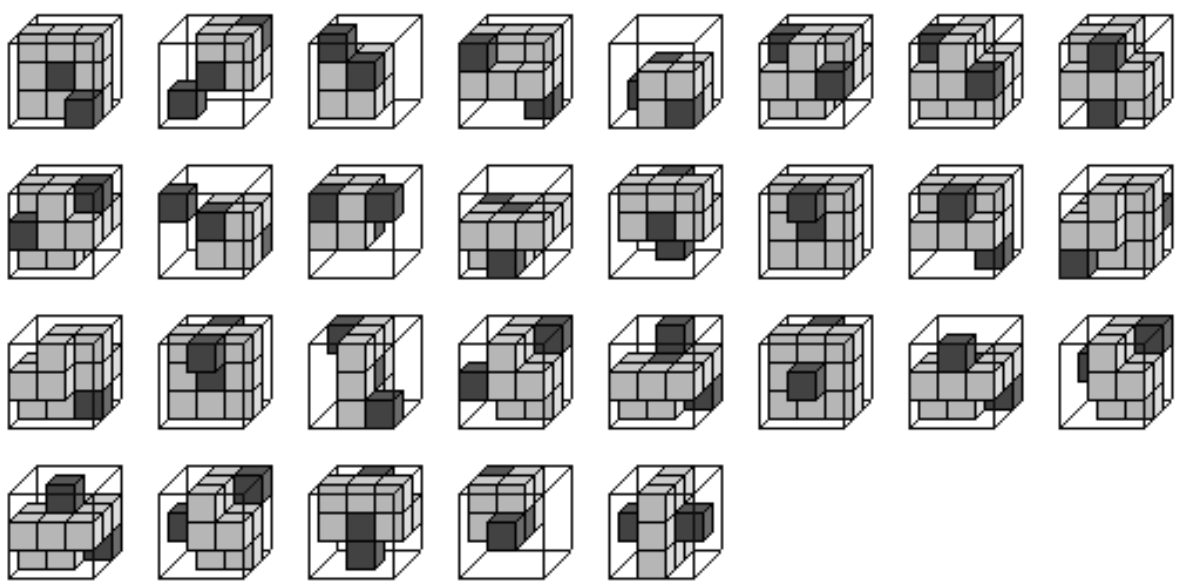

Fig. 13. Shape detectors for space curves "Curv26"

\subsection{Erosion with Shape Primitives as Conditions: Skeletonization}

The main application of the shape detectors is that they can be used as conditions for topology preserving erosion, or skeletonization. The method of chapter 2, iterating over an image $\mathrm{X}_{3}$ with the erosion mask "Erode26cct" of figure 3 and the sets of figure 12 (Surf26) and 13 (Curv26), yields a skeleton. Erode26cct erodes surfaces from volumes (it hits only on the core of the volumes, not on their boundaries). The set Curv26 prevents the erosion of surfaces (the masks only hit on the core of the surface, not on the surface boundaries). The set Surf26 prevents the erosion of curves (the masks only hit on the kernel of the curves). Consequently, as volumes, surfaces and curves cannot be eroded from their kernels, they are eroded from their boundaries. So only closed surfaces and curves will remain.

To prevent the erosion of boundaries, from the set Curv26 a new set Curv26e is made that contains all surface edge situations. Similarly, a set Curve26e can be made from the set CURV26 containing all curve end situations. A simple procedure can be followed. A surface extends in 4 directions. Systematically one and two directions can be disabled by setting them to background. A curve extends in two directions, one of which can be disabled by setting it to background. This yields 87 unique configurations for the surface edges and 3 possibilities for curve ends.

A final remark should be made on the detection of two element thick structures. They cannot be detected properly in a $3^{\mathrm{N}}$ neighbourhood. To circumvent this, the recursive neighbourhood must be used to detect foreground-background changes. However, only for the Surf 26 and Curv26 sets and not for the erosion mask and the Surf26e and Curv26e masks!

A variety in skeletons can now be made (see figures 14 and 15) 

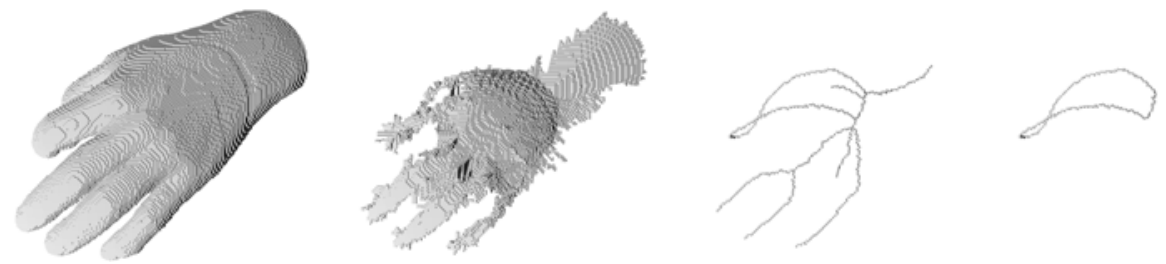

Fig. 14. Ofiginal ${ }^{1}$, surface skeleton, curve skeleton and skeleton without curve ends

The set $\{$ Erode26ct, Surf26, Surf26e and Curv26\} was used to obtain the surface skeleton. The set \{Erode26ct, Surf26, Curv26 and Curv26e\} was used to obtain the curve skeleton. The set \{Erode26cct, Surf26, Curv26\} was used to obtain the last skeleton of figure 14. All masks from all sets can be used in parallel. However, the local situation in the image determines which masks are active. E.g. if the image does not contain voluminous and surface objects anymore, only the Curv26 set is active, preventing the breaking of curves that are eroded from their ends.

An important observation is that the masks that prevent the breaking of the topology for a certain intrinsic dimension $\widetilde{N}$, are the erosion conditions for objects with that intrinsic dimension $\widetilde{N}$. Consequently, the mask Erode26cct prevents breaking the topology of a (flat) volume and the set Surf26 incorporate the erosion conditions and metric (!) for the curved surfaces. These metrics, due to the mask Erode26cct and set Surf26, are not Euclidean, which results in deviations of the skeleton from the medial axes of the original object. When the skeleton is needed for measurement purposes, the sets Surf26, Surf26e, Curv26, Curv26e cannot be used in parallel, but must be applied sequentially in the following way. A constrained Euclidean distance transform [19] should be performed on the original volume, where-after the surface skeleton should be made, eroding voxels in an order that depends on their distance to the volume boundary. Subsequently, on the surface skeleton, a constrained distance transform should be made, after which the curve skeleton should be made eroding voxels in the order of their distance to the surface edge.

Figure 15 shows the use of a curve skeleton \{Erode26cct, Surf26, Curv26\} in pathfinding through a maze. Start and endpoint are inserted in image $Z^{3}$ and using an extra mask anchor the skeleton onto those two points.

For Robot Soccer [20] a similar anchor skeleton was used in the "Dutch Team" to quickly plan the collision free path from start to goal of an attacker robot. The image $\mathrm{X}_{3}$ represented the universe of the robot with two place dimensions (x,y) and one time dimension $(\mathrm{t})$. Manipulating both the erosion metric and the mask sets made it possible to prevent the time from running backwards and setting a speed maximum for the robot. If the orientation of the robot should be taken into account also, the problem should be solved in $\mathrm{X}_{4}(\mathrm{x}, \mathrm{y}, \theta, \mathrm{t})$.

${ }^{1}$ The real original image (from Toriwaki) contained two holes, giving rise to two closed surfaces at the skeleton near the wrist. We closed the holes beforehand, for didactical reasons. 

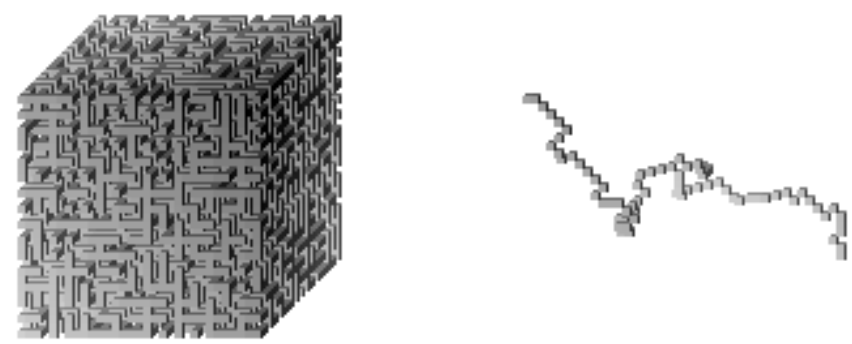

Fig. 15. An anchor skeleton is used to solve a path finding problem

\subsection{Verification}

The method described in this paper lends itself very well for extension into higher dimensions. Provided the approach is valid. This was verified by comparing the outcome of the masks for $\mathrm{X}_{2}$ with the Hilditch [21] skeleton and outcome of the masks for $\mathrm{X}_{3}$ with the approaches of Lobregt 22] / Toriwaki [23] and Malandain 24]. They were checked by comparing and found to have an identical outcome in all $2^{9}$ and $2^{27}$ possibilities of the $3^{2}$ and $3^{3}$ neighbourhood. Note, however, that in all three methods only the curve skeleton could directly be made, as the conditions can not be split over surface and a space curve sets. Hence, no accurate Euclidean variants could be established with those methods.

To prepare for verification in $\mathrm{X}_{4}$ we extended the Euler count (Lobregt) / cluster count (Toriwaki) method to 4D 25. As the Euler-sum 26 in 4D is always zero, we solved this by embedding $4 \mathrm{D}$ objects in $\mathrm{X}_{5}$ to calculate the 5D Euler number. This number changes due to 3 events: 1D tunnels $(\widetilde{N}=1), 2 \mathrm{D}$ tunnels $(\widetilde{\mathrm{N}}=2)$ and 3D tunnels $(\widetilde{N}=3)$. As the latter cannot occur in $\mathrm{X}_{4}$, with Euler-, and fore- and background cluster counting, the number of equations just equals the number of unknowns and a topology change can be detected. As the Euler sum applied to objects in $\mathrm{X}_{5}$ counts also the possible 3D tunnels, fore- and background cluster counting is now not enough to detect the cause of change in the Euler sum. Consequently, as fore- and background cluster counting is all we can do in any dimension, the Euler/cluster count approach is only applicable in $\mathrm{X}_{3}$ and (using $\mathrm{X}_{5}$ ) in $\mathrm{X}_{4}$, making $\mathrm{X}_{4}$ the limit.

We generated test images in $X_{4}$ based on circular closed objects of various intrinsic dimension, such as 4D equivalents of hollow balls, circles, etc. and contaminated them with foreground noise. The 4D skeleton showed to be robust. 


\section{Shape Primitives in $X_{4}$}

The methods described in this paper extend straightforward to $X_{N}$, especially for the basic operations such as erosion, dilation, contour extraction, propagation etc. involving only a few masks. To get an impression how the interaction between foreground and background shapes primitives and their intrinsic dimension works out in $\mathrm{X}_{4}$ we show in figure 16 how the paradox of foreground and background shape primitives works out. We define:

Topology breaking of a foreground shape primitive of dimension $\widetilde{\mathrm{N}}$ is done by a background shape primitive of intrinsic dimension $\mathrm{N}-\widetilde{\mathrm{N}}$ (and vice versa).

For example, a space curve cannot be broken by a point. As we only have foreground and background, the background point must be connected to other background. This cannot be a line, because the foreground curve leaves two degrees of freedom c.q. intrinsic dimensions open. For a simplified view on the matter in $\mathrm{X}_{4}$, we assumed in figure 16 lowest connectivity for fore- and background and the existence of flat objects only. Figure 16 shows five sets of shape primitives: Hyper-volume threatened to be broken by a point, volume threatened by a line, plane threatened by a plane, line threatened by a volume an point threatened by a hyper-volume. It indicates that because we consider the topology breaking as the union of a number of individual events, we do not run into the problem of the Euler-Cluster count method, that cannot make a distinction between the occurrences that lead to a change in the Euler number.

On top of the paradox of the shape primitives we find the connectivity paradox. Table 1 indicates the relation between a dimension, its shape primitives and their associated connectivities. We distinguish CE: Connectivity to the central Element, $\mathrm{NN}$ : number of neighbours required for this shape primitive, RC: Recursive connectivity.

The generation of the various sets follows the same lines as was done for $X_{3}$. A shape primitive of intrinsic dimension $\widetilde{N}$ is made by encircling the central element over the positions CE, by shape primitives of intrinsic dimension $\widetilde{N}-1$ and connectivity RC. This is a recursive procedure. E.g. a foreground hyper-volume is made by encircling it by a closed foreground surface, etc. 


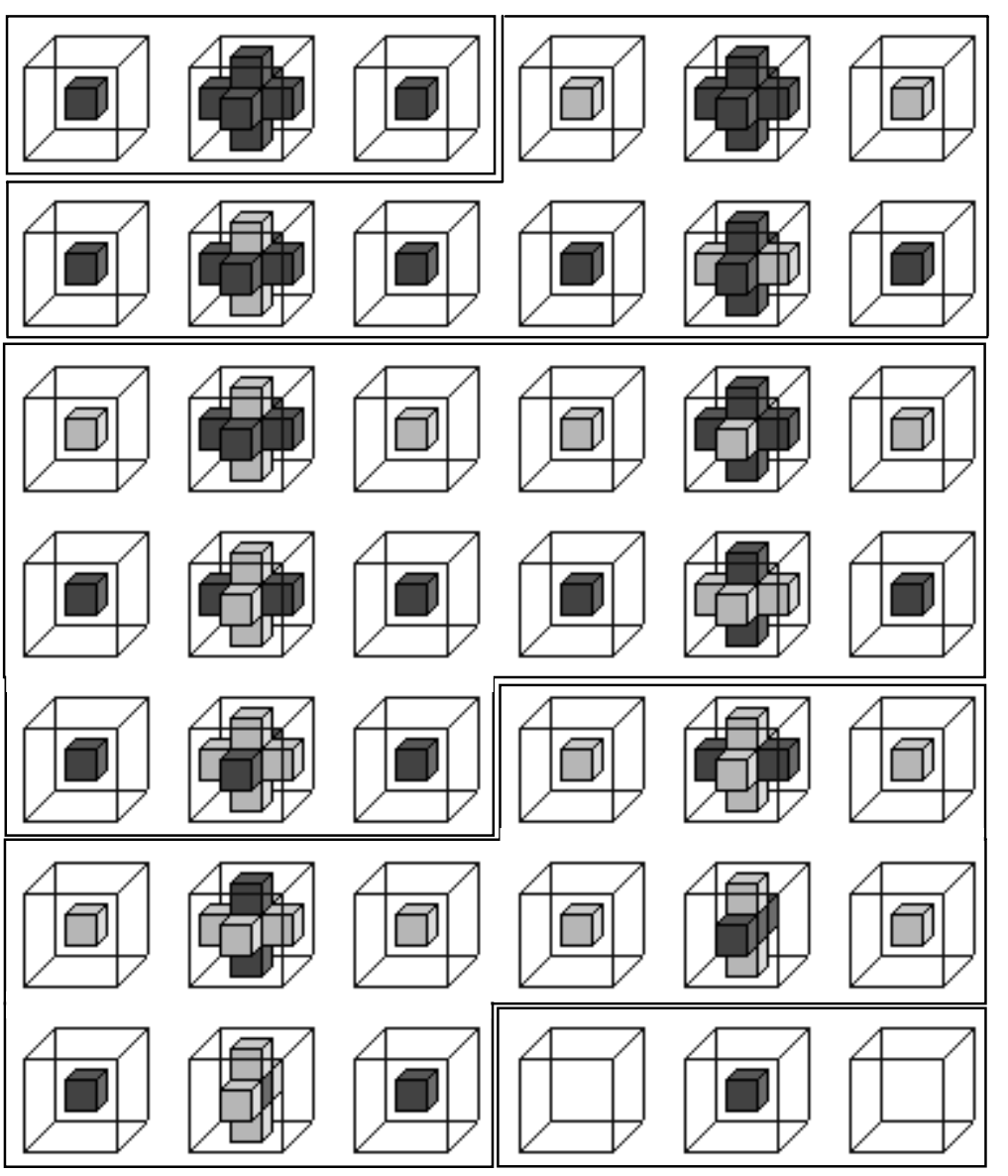

Fig. 16. A simplified view on the shape primitive paradox in $X_{4}$

Table 1. Dimension, intrinsic dimension and connectivity for various shapes

\begin{tabular}{|c|l|c|c|c|l|c|c|c|}
\hline $\mathrm{N}, \widetilde{\mathrm{N}}$ & Foreground & $\mathrm{C}$ & $\mathrm{N}$ & $\mathrm{R}$ & Background & $\mathrm{C}$ & $\mathrm{N}$ & $\mathrm{R}$ \\
& shape primitive & $\mathrm{E}$ & $\mathrm{E}$ & $\mathrm{C}$ & shape primitive & $\mathrm{V}$ & $\mathrm{E}$ & $\mathrm{C}$ \\
\hline$(2,2)$ & Flat surface & 4 & 4 & - & Point & - & - & - \\
\hline$(2,1)$ & Curve & 8 & 2 & - & Curve & 4 & 2 & - \\
\hline$(3,3)$ & Flat volume & 6 & 6 & - & Point & - & - & - \\
\hline$(3,2)$ & Curved surface & 18 & $\geq 4$ & 26 & Space curve & 6 & 2 & 6 \\
\hline$(3,1)$ & Space curve & 26 & 2 & - & Curved surface & 18 & $\geq 4$ & 6 \\
\hline$(4,4)$ & Flat hyper-volume & 8 & 8 & - & Point & - & - & - \\
\hline$(4,3)$ & Curved volume & 32 & $\geq 6$ & 64 & Space curve & 80 & 2 & 8 \\
\hline$(4,2)$ & Curved surface & 64 & $\geq 4$ & 80 & Curved surface & 64 & $\geq 4$ & 8 \\
\hline$(4,1)$ & Space curve & 80 & 2 & - & Curved volume & 32 & $\geq 6$ & 8 \\
\hline
\end{tabular}




\section{Conclusions}

In this paper we described a framework for mathematical morphology or cellular logic operations that can in principle be extended to $\mathrm{N}$ dimensional (hyper) cubic tessellated images. We focussed on the constructions of shape primitives that can be used to detect shape properties locally in the image. Shape primitives are grouped in intrinsic dimension sets, such as volume, surface and curve primitives. The shape properties can be used to identify parts (e.g. all surface edges) and hence help to segment the image. By tagging each primitive with a certain contribution, measurements that are more accurate can be done. Finally, they can be used as topology breaking conditions for the generation of skeletons. Because they are the union of a sub-set of conditions, this enables the smooth creation of various skeleton variants. The number of shape primitives is remarkably low in $\mathrm{X}_{3}$. A skeleton of a $200^{3}$ image takes about 5 minutes.

The method is extendable to higher dimensions. This is straightforward for simple morphological operations, however, not simple for shape primitives. A method was presented to automatically generate the shape primitives in $\mathrm{X}_{3}$ and its extension to $\mathrm{X}_{4}$ was indicated. The verification of the generated $\mathrm{X}_{4}$ primitives comparing it with a skeleton for $\mathrm{X}_{4}$ based on an Euler count / cluster count method, is ongoing research [27].

\section{References}

1 Jonker P.P. (1992) Morphological Image Processing: Architecture and VLSI design. Kluwer Technische Boeken BV, Deventer, The Netherlands. ISBN 90201-2766-7

2 Golay M.J.E. (1969) Hexagonal Parallel pattern transformations. IEEE transactions on computers C-18, 8:733-740

3 Preston K. Jr. (1970) Feature extraction by golay hexagonal pattern transforms. IEEE Symposium on feature extraction and selection in pattern recognition, Argonne, III

4 Serra J. (1982) Image analysis and mathematical morphology. Academic Press, Inc. London.

5 Serra J. (1988) Image analysis and mathematical morphology. Volume II: Theoretical Advances. Academic Press, Inc. London.

6 Giardina, C.R. (1988) Morphological methods in image and signal processing. Prentice Hall, Englewood Cliffs-NJ. ISBN 0-13601295-7

7. Heijmans H.J.A.M., (1994) Morphological Image Operators. Academic Press. Boston

8 Soille P., (1999) Morphological Image Analysis, Springer Verlag, Berlin

9 Jones R., and Svalbe, I.D., (1994) Basis Algorithms in Mathematical Morphology, Advances in Electronics and Electron Physics, Vol.89, Academic Press, Inc. ISBN 0-12-014731-9

10 Matheron, G. (1975) Random Sets and Integral geometry. Wiley, New York 
11 McClusky E.J. (1965) Introduction to the theory of Boolean functions, mcCrawHill, New York

12 Rosenfeld A., Pfalts J.L. (1966) Sequential operations in Digital Image Processing. Journal of the ACM, 471-494.

13 Coxeter H.S.M. (1974) Regular Polytopes ( ${ }^{\text {rd }}$ edition) Dover Publications, Inc. New York, ISBN 0-486-61480-8

14 Jonker P.P., Vossepoel A.M. (1994) Connectivity in high dimensional images, Proc. MVA 94, IAPR Workshop on Machine Vision Applications, Kawasaki, Japan, Dec.13-15.

15 P. Alexandroff (1961)Elementary concepts of topology. Dover Publications, Inc. New York, ISDN 0-486-60747-X

16 Jonker P.P., Vossepoel A.M. (1995) On skeletonization algorithms for 2, 3 .. N dimensional images, in: D. Dori, A. Bruckstein (eds.), Shape, Structure and Pattern Recognition, World Scientific, Singapore, 71-80. ISBN 981-02-2239-4

17 Brayton R.K., Hachtel G.D., McMullen C.T., Sangiovanni-Vincetelli A.L. (1984) Logic minimization algorithms for VLSI synthesis. Kluwer, Dordrecht / Boston

18 Mullikin, J. J.C., Verbeek P.W.(1993), Surface area estimation of digitized planes, BioImaging, vol. 1, no. 1, 6-16.

19 Verwer B.J.H. (1991), Local distances for distance transformations in two and three dimensions, Pattern Recognition Letters, vol. 12, no. 11, 1991, 671-682.

20 www. robocup.org, www.robocup.nl

21 Hilditch C.J (1969) Linear Skeletons from square cupboards. in B. Meltzer and D. Mitchie (eds.) Machine Intelligence Vol. 4. Edinburgh: University Press, 404-420.

22 Lobregt S., Verbeek P.W, Groen F.C.A, (1980) Three dimensional skeletonization: Principle and algorithm IEEE Trans. Patt. Anal. Machine Intell. vol. 2, pp. 75-77

23 Toriwaki J., Yokoi S., T. Yonekura T, Fukumura F (1982) Topological properties and topological-preserving transformation of a three dimensional binary picture. in Proc. Int. Conf. Patt. Recogn., Munich 414-419

24 Malandain C, Bertrand B (1992) Fast Characterixation of 3D Simple Points. Proceedings of the 11th ICPR, Vol III, The Hague.

25 Jonker P.P., Vermeij O. (1996), On skeletonization in 4D images, in: P. Perner, P. Wang, A. Rosenfeld (eds.), Advances in Structural and Syntactical Pattern Recognition, Lecture Notes in Computer Science, vol. 1121, Springer Verlag, Berlin, 79-89.

26 Hilbert D, Cohn-Vossen S (1932) Anschauliche Geometrie, Springer Verlag, Berlin.

27 Tools for generating, manipulating and executing mask sets for 2, 3 and 4 dimensional images can be found on ftp.ph.tn.tudelft.nl/pub/clop 